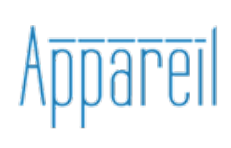

Appareil

3| 2009

Concerts publics et formes de la sensibilité musicale

\title{
Concerts des Nations
}

Regards sur la naissance du Domaine musical et celle du Concert spirituel

Sarah Barbedette

\section{(2) OpenEdition \\ Journals}

Édition électronique

URL : http://journals.openedition.org/appareil/817

DOI : 10.4000/appareil.817

ISSN : 2101-0714

Éditeur

MSH Paris Nord

Référence électronique

Sarah Barbedette, "Concerts des Nations », Appareil [En ligne], 3 | 2009, mis en ligne le 29 mai 2009, consulté le 30 juillet 2020. URL : http://journals.openedition.org/appareil/817 ; DOI : https://doi.org/ 10.4000/appareil.817

Ce document a été généré automatiquement le 30 juillet 2020.

\section{(c) (i) () $\Theta$}

Appareil est mis à disposition selon les termes de la Licence Creative Commons Attribution - Pas d'Utilisation Commerciale - Pas de Modification 4.0 International. 


\title{
Concerts des Nations
}

\author{
Regards sur la naissance du Domaine musical et celle du Concert \\ spirituel
}

\section{Sarah Barbedette}

\section{Images d'un prisme}

1 À s'interroger sur la naissance du concert, toujours recommencée, sur la venue au monde d'organisations périodiques de concerts publics, grande est la tentation de vouloir démêler une part d'invariance de celle de contingence, ou du moins de chercher à faire émerger quelque ossature reconnaissable. En de tels cas cependant,

il faut une conjonction d'une multitude de faits et de choses (le hasard et l'arbitraire mis à part) pour constituer un événement. Ces faits se préparent, viennent de loin, se rejoignent, s'entrecroisent, se contredisent, se heurtent même, pour aboutir, en certains cas privilégiés, à quelque chose qui s'appelle la réussite, à quelque chose qui peut être classé sur le plan d'une signification historique' ${ }^{1}$.

2 Ces mots de Pierre Souvtchinsky, en forme de salut aux dix ans d'existence du Domaine musical, posent la complexité de la naissance de telles entreprises. Si l'histoire en a distingué certaines, chacune de celles-ci n'en relève pas moins de ces conjonctions multiples propres aux événements, qui valent régulièrement de s'enfoncer dans les méandres des particularismes et d'échouer à dresser une typologie, ou de renoncer à ébaucher un archétype.

3 Ne serait-il pas possible pour autant, au cœur de ces mille et un faisceaux convergents et contradictoires, de poser face-à-face deux organisations historiquement différenciées et de les installer dans une situation dialogique? Si l'une comme l'autre, nonobstant l'ampleur des années qui les séparent, répondent à l'image d'«une entreprise musicale utile et importante qui fit époque dans les annales de l'art ${ }^{2}$ ", réussira-t-on pour autant à engager plus avant un va-et-vient comparatiste, un dialogue dont le miroitement ne soit par trop artificiel?

4 La technique du parallélisme tient à beaucoup d'égards d'un « forçage » théorique. Mais considérer deux institutions musicales comme les deux «bases» d'une figure possible 
du concert est un parti pris que la géométrie spatiale n'entache ni ne relève : il s'agit plutôt de concevoir un prisme fictif pour installer l'une et l'autre dans un dialogue frontal, de les penser sous une figure double afin d'atténuer l'identité de chacune, et de voir aussi, par mouvement contraire, se relever leurs originalités propres.

Le Domaine musical, dont on a déjà cité le nom, se distingue par sa position "tuilée ", charnière dans l'histoire du concert, qui tient tout à la fois de la clôture d'un cycle avec le chant du cygne des Salons - et du renouveau absolu de la vie musicale, au sortir de la guerre. "Clôture » ou "renouveau » ne pouvant se définir sans un regard sur l'histoire passée du concert, le Concert spirituel s'est imposé comme un vis-à-vis essentiel. Première institution de concerts publics en France, le Concert spirituel a eu une activité de plus de soixante années sous l'Ancien Régime (1725-1790) quand le Domaine musical a lui été actif de 1953 à 1973, marquant pendant deux décennies le cœur du Xxe siècle 3 .

\section{Naissance du Domaine musical}

6 Conçu avec Pierre Souvtchinsky, le "Domaine-musical-de-Pierre-Boulez » - ainsi que beaucoup le nomment - naît au milieu du xx siècle sous l'aile de Jean-Louis Barrault et de Madeleine Renaud. Pierre Boulez est alors directeur de la musique de scène de la Compagnie depuis huit ans, et n'a pas grande difficulté à convaincre Jean-Louis Barrault de l'urgence de faire entendre la musique nouvelle. Pris en charge par le comédien, les concerts s'intègrent à la saison du Théâtre Marigny et bénéficient immédiatement du renom de la troupe. Après un an d'activité menée sous le nom de Concerts du Petit Marigny (saison 1953-1954), Pierre Boulez, aidé du musicologue Pierre Souvtchinsky, de Suzanne Tézenas et de Jean-Louis Barrault, dépose les statuts de l'association du Domaine musical à la préfecture de Paris ${ }^{4}$. Barrault continue d'héberger les concerts dans son théâtre (les jours où il n'y a pas de représentation), mais n'en supporte plus les frais exorbitants, tandis que Suzanne Tézenas et Pierre Souvtchinsky mettent à contribution leurs carnets d'adresses et leurs relations tout à la fois pour financer la saison et solliciter la venue d'insignes personnalités ${ }^{5}$.

7 La programmation s'organise en trois plans et Boulez veille à ce qu'en son sein, la musique sérielle soit bien représentée et bien jouée. Il découvre alors une chronophagie inhérente à la tâche : " présenter comme il faut des concerts comme cela ${ }^{6}$ » est un crèvecœur puisque seul un musicien peut réellement mener à bien le travail de conception, et que ce métier d'organisateur lui retire son instrument des mains, quand ce n'est la plume. Boulez n'accuse le poids de ce travail qu'à la fin de la saison 1953-1954, charge sans commune mesure avec celle des quelques concerts qu'il avait pu organiser auparavant chez Suzanne Tézenas. Au nombre de ces concerts donnés dans le Salon de la rue Octave-Feuillet plusieurs années avant la création du Domaine musical, figurait notamment la première audition française des Sonatas and Interludes pour piano préparé de John Cage, en 1949 ; mais - si arrosé de cognac que fut le piano ${ }^{7}$ - de tels concerts n'engageaient pas les effectifs réunis au Petit Marigny et se réduisaient le plus souvent à un récital pour piano seul, limitant ainsi les complications matérielles y afférentes.

Chez Suzanne Tézenas qui était très liée aux écrivains de la nrf, et du fait de son travail auprès de Jean-Louis Barrault, Boulez est entouré d'une intelligentsia littéraire que les concerts du Petit Marigny adopteront sans contrainte pour s'y lover: "Sur cette nouvelle scène, prendra place une activité théâtrale sur laquelle Jean-Louis Barrault a 
déjà donné des précisions [...] Une association culturelle en formation - pour ne point négliger cet aspect de la connaissance et de la recherche - se propose d'adjoindre au domaine proprement littéraire, des manifestations réservées à la création musicale " écrit Boulez pour annoncer la première série de concerts donnée dans la toute nouvelle petite salle du Théâtre Marigny8. Présenté à la presse comme un « laboratoire », le tant attendu « théâtre d'essai » est inauguré par la musique, le 13 janvier 1954.

\section{Naissance du Concert spirituel}

Le Concert spirituel, première institution de concerts publics et payants en France, est créé en 1725 par Anne Danican Philidor (1681-1728). Hautbois de l'Écurie, de la Chapelle royale et de la Chambre du roi ${ }^{9}$, celui-ci obtient, moyennant une redevance annuelle, le privilège de donner des concerts publics « les jours où il n'y aura point de spectacle, comme les trois semaines de Pasques, la Pentecôte, la Toussaint, Noël et touttes les festes de Vierges et veilles ${ }^{10}$ ». Marque de la bienveillance royale à l'égard de la nouvelle institution, mais aussi de sa nature déclarée spirituelle, l'annonce est faite par le Mercure de France en mars 1725, non dans la rubrique «spectacles » où l'on peut lire l'actualité concernant l'Opéra, mais dans la rubrique «Nouvelles de la cour »... Ambivalence constitutive du Concert spirituel : il convient aux «nouvelles de la cour », est né du privilège royal, diffuse censément un répertoire spirituel et n'entre pas dans la catégorie des spectacles... Bien que l'Académie royale de musique comptabilise dans ses recettes la redevance due par le Concert spirituel, et aille jusqu'à en assurer la direction entre 1734 et 1748 .

Descendant d'une famille de musiciens estimés et protégés par le roi depuis plusieurs générations, ordinaire du roi pendant les dernières années du règne de Louis XIV, Philidor ayant obtenu le privilège royal d'organiser des concerts publics à Paris, s'associe - pour des raisons essentiellement financières - avec Delannoy afin d'assurer la direction du Concert spirituel (1725-1728). Le Concert spirituel se voit alors destiner le grand salon du château des Tuileries, ou salle des Cent-Suisses, réaménagée pour l'occasion, et à laquelle est rapidement reconnue une fonction de «laboratoire musical ${ }^{11}$ » de l'Europe - réputation de " laboratoire » dont les espoirs exprimés comme un leitmotiv par Leopold Mozart laissent encore la trace ${ }^{12}$. Quarante ans après la naissance du Concert spirituel, beaucoup soulignent cependant le discrédit dans lequel il est tombé : Charles Burney ne ménage pas ses critiques et, dans son étude menée au début $\mathrm{du} \mathrm{xx}^{\mathrm{e}}$ siècle, Michel Brenet juge quelque peu suspect l'enthousiasme d'un Bricaire de la Dixmerie ${ }^{13}$ rappelant, en contrepoint des frilosités du concert public, les vues éclairées et l'action au plus haut niveau musical menée par La Pouplinière. Cependant le Concert spirituel naît avant le Salon de La Pouplinière et ses premières années, malheureusement peu documentées, restent celles de la première ouverture à la musique non-dramatique dans un cadre public et payant à Paris.

11 Avant 1725, la vie de Philidor est un mystère. On dit - chaque article sur ce point reproduit avec une scrupuleuse fidélité le conditionnel - qu'il aurait été administrateur des grandes fêtes de la duchesse du Maine de Sceaux ${ }^{14}$. Ces "divertissemens tantôt comiques, tantôt sérieux, dont les paroles \& la musique étoient des plus grands Maîtres de l'art, \& toujours exécutez par les plus fameux Acteurs et Danseurs du Théâtre, avec des habits faits exprès, \& des décorations pour le sujet, où rien n'étoit épargné ${ }^{15}$ ", adressés à la plus haute noblesse de cour, se distinguaient par leur auditoire éclairé, 
d'intellectuels, d'écrivains ou de jeunes philosophes (Voltaire). Il se pourrait en réalité qu'une telle affirmation trouve son origine dans la confusion de deux informations distinctes : le fait que Mouret, deuxième directeur du Concert spirituel (1728-1733), a bien été Intendant des Nuits de Sceaux tandis que Philidor, lui, était l'auteur de pièces composées pour ces mêmes Nuits de Sceaux - et encore cela concerne-t-il une période antérieure à l'année $1715^{16}$. L'autre emploi que Philidor aurait occupé durant ces années de «vacance » est celui de Surintendant de la musique du Prince de Conti, ce qui paraît moins douteux dans la mesure où le fait est mentionné par Jean-Benjamin de La Borde dans son Essai sur la musique paru en $1780^{17}$. Il n'en reste pas moins qu'après la mort de Louis XIV, la vie de Philidor est essentiellement marquée par le sceau du vide documentair ${ }^{18}$, renvoyant l'attention du chercheur aux délinéaments de son existence. Ces dix années (1715-1725) succèdent à une période de guerres qui a rendu la France exsangue; la cour déserte Versailles pour Paris, et s'effiloche tout ce que seule la présence de Louis XIV maintenait en des mailles qui ne demandaient qu'à lâcher. C'est alors un mouvement de reconquête de la capitale qui se dessine, la volonté de tourner une page, l'urgence de réinventer. Les salons et sociétés de concerts privés s'organisent, accueillent les musiciens $\mathrm{du}$ roi, et font entendre une musique instrumentale que l'inanité des privilèges royaux obtenus par Lully et transmis à ses successeurs limite aux exécutions de la Chambre et de la Chapelle du roi. Le mouvement général tend alors à la multiplication des concerts (fussent-ils privés), et à une réinscription la vie culturelle dans Paris. L'ouverture du Concert spirituel, dix ans après la mort du Roi-Soleil, suit exactement ce mouvement, qui change la donne pour les auditeurs, mais aussi pour les compositeurs.

12 Ces deux images brèves, partielles, asymétriques et non parallèles auront peut-être suffi à dire toute la différence qui règne entre les deux entreprises que sont le Domaine musical et le Concert spirituel. Toutefois, si l'on tente le "montage parallèle » de certaines séquences, des traces de correspondances se font jour, laissant quelques questionnements se former à l'émergence de lignes de crêtes. Des dénominateurs communs apparaissent malgré tout, tissant entre les deux concerts une communauté de faits, de logiques, d'agencements.

\section{Flux}

Dans les deux cas de figure observés, un musicien, chargé de l'intendance de concerts privés, se lance dans l'organisation de concerts publics (il en assure la direction mais veille à être secondé pour la partie proprement financière). Concert spirituel et Domaine musical semblent alors l'expression mise en abyme du mouvement historique marquant l'évolution du concert, qui consiste à germer dans un cadre privé pour ensuite s'épanouir dans la sphère publique. Après la première soirée donnée dans le «laboratoire» de Barrault, le journal Arts prenait ainsi la peine de remarquer: «Au Petit-Marigny, en effet, l'organisateur est un compositeur - ce qui est un fait assez nouveau - un musicien qui a maintes fois donné des preuves de son indépendance, de sa fermeté au point de vue esthétique ${ }^{19}$ ». Un musicien qui saisisse, dans l'état de la musique qui l'entoure, un manque, la nécessité d'une intervention. Faire entendre la 
musique de l'École de Vienne et les jeunes compositeurs sériels, ou défaire la musique de son assujettissement au drame lyrique auront été des révolutions inéluctables menées par et pour ceux qui font la musique, grâce à l'argent et au snobisme d'une société mondaine, et sans afficher haut l'étendard de l'esthétique revendiquée. Il s'agit bien plutôt de se mouvoir dans des cercles intellectuels et artistiques larges que de s'étioler dans un milieu purement musical.

Annonçant que "le sieur Philidor fit exécuter..." les œuvres choisies pour chaque concert donné dans la salle des Cent-Suisses, Le Mercure de France avait soin de rappeler quelle en était la direction artistique, tandis que les concerts du Domaine n'avaient souvent d'autre nom que celui de «Concerts Boulez». Bien que le souci de Pierre Boulez ait été de donner à entendre la musique nouvelle et celle des «aïeux privilégiés », cette ligne directrice profonde n'a jamais été présentée comme telle dans les premiers programmes, qui mettaient essentiellement en valeur une idée de comparatisme, exercée à travers la variété des effectifs sonores et la perspective de plusieurs siècles de musique ${ }^{20}$. Que Boulez ait tenu à choisir un nom « tellement neutre, que les gens ne pouvaient pas savoir à quoi se raccrocher ${ }^{21}$ ", quand tout le monde qualifiait avec évidence les concerts du Domaine de concerts « dodéca » n'est pas sans contradiction. Choisir ce titre - dont on a trop peu relevé les qualités d'oxymore - est à n'en pas douter, sinon immédiatement un gage de longévité, tout au moins un gage de liberté.

D'une manière quelque peu différente, l'entreprise de Philidor, théoriquement autorisée dans la mesure où elle ne contrevenait pas au calendrier liturgique, était annoncée, elle, comme organisation de concerts de «musique spirituelle». Or, toute étude menée autour du Concert spirituel oscille en permanence entre cette affirmation conditionnelle d'un répertoire spirituel et l'inauguration de fait par un programme qui « commença par une suite d'Airs de violons de M. de la Lande, d'un caprice du même Auteur, \& de son Confitebor. On joüa après la nuit de Noël, Concerto de Correlli, ajoute le Mercure de France, \& le Concert finit par le Cantate Domino ${ }^{22}$ ». Difficile de savoir où se situe la feinte soumission au répertoire spirituel quand, dès le mois de mars 1725 , ces "Concerts composez de musique spirituelle" sont annoncés dans une proposition conclue par ces mots : « lesquels [concerts] sont composez de Motets à grands Chœurs, \& de Simphonies françoises et Italiennes des meilleurs Auteurs » - où toute l'ambiguïté est englobée par la syntaxe dans une seule et même phrase.

En somme, ces deux entreprises tiennent à garder les coudées franches d'un point de vue programmatique et se dégagent de toute soumission à un intitulé par des choix extrêmement pragmatiques, même si de facto le nom de l'initiateur suffit souvent à déduire un engagement sans ambiguïté. D'une certaine manière, la personnalisation de ces programmes participe du passage du privé au public qu'on a décrit plus haut: le Concert spirituel est né parce que Philidor avait œuvré au service du roi puis tenté probablement l'intendance de quelque concert privé. Le Domaine musical est né parce que Boulez a claqué la porte du Conservatoire, parce qu'il a travaillé avec Barrault, qu'il a rencontré Suzanne Tézenas... Les précédents à la naissance de chaque concert se trouvent dans l'exercice d'un métier mené en marge de la pratique musicale: côtoiement de cercles littéraires et artistiques, déplacements légers, sentiers voisins... Chacun des deux compositeurs aura poursuivi à travers la création du Concert et du Domaine, une œuvre dont aucune borne ne peut être assimilée avec lesdits concerts... Où l'on retrouve toujours le faisceau d'incernables prémisses dessiné par Pierre 
Souvtchinsky. Mais on voudrait souligner aussi, outre la continuité imputable à la permanence du travail d'un homme (lequel dans chaque cas transmettra sa charge de direction), le fait que la naissance du concert se situe à un endroit médian, qu'elle n'est pas une origine mais prise dans un développement qui la dépasse, qu'il y a un avant et un après difficilement cernables, et que la naissance du concert reste un entre-deux. Que la naissance du concert tient du rhizome et non de la racine, pour reprendre l'idée deleuzienne, en ce sens que par tous les pans qui la mènent, l'activité du concert implique celles qui ont été engagées auparavant et celles qui suivront. Ainsi certains auditeurs du Domaine musical assimilent-ils le concert Cage de 1949 avec les concerts du Domaine, ou l'activité du Domaine avec celle de l'Ircam. Il est plus difficile aujourd'hui d'établir le lien qui peut exister, du fait des emplois de Philidor, entre le concert des Mélophilètes (placé sous les bons auspices du Prince de Conti) et le Concert spirituel. On peut en revanche parler du concert effectif comme déplacement des activités, dans la mesure où le répertoire instrumental était déjà en partie joué dans un cadre privé, et par des musiciens issus de formations en exercice (l'Orchestre de l'Académie royale de musique au xviII ${ }^{\mathrm{e}}$ siècle, l'Orchestre national et l'Orchestre de l'Opéra de Paris au $\mathrm{xx}^{\mathrm{e}}$ siècle, formations royale ou d'État). Ce sont donc les mêmes musiciens qui jouent avant et après la naissance du concert, un répertoire qui ne vient pas précisément de naître, mais auquel la publicité de l'événement donnera un élan et des moyens nouveaux. Le concert tire en somme, dans un pas de côté, les fils de tissages préexistants et co-existants, s'inscrivant tout à la fois en dissidence esthétique et dans une continuité de fait.

17 On vient de souligner que la naissance du concert n'est pas un point d'origine, que l'idée même de bornes est immédiatement battue en brèche pour ce qui touche au répertoire, et que la porosité des sphères privée et publique œuvre à l'échelle de l'histoire du concert tout comme elle régit chaque soir les engagements des musiciens. Dès lors aiguillée par une question qui prend forme - à quoi tient le sentiment profond d'avoir affaire, dans chacun de ces deux cas, à une entreprise essentiellement rétive à la notion de frontière? - on peut remarquer, même s'il ne s'agit que d'un petit pas supplémentaire au cœur d'une problématique qui ne sera pas épuisée dans ces lignes, que la notion de frontière géographique n'existe pour le Domaine musical ou le Concert spirituel qu'à la condition d'être transgressée.

\section{«Europe »}

18 Au début du xvIII ${ }^{e}$ siècle, alors qu'on mène le Concert spirituel sur les fonds baptismaux, certains concerts privés défendent la musique italienne (le concert italien des Amateurs), d'autres la musique française (le concert français des Mélophilètes) ${ }^{23}$, et l'on caractérise couramment un concert occasionnel par ses composantes italienne ou française ${ }^{24} . .$. La nécessité qui s'exprime alors est d'ouvrir un concert qui s'affranchisse d'un parti exclusivement lié à telle ou telle musique... Le Mercure spécifie ainsi, dès 1725, que musique française et musique italienne seraient interprétées au Concert spirituel et, quarante ans plus tard, Bricaire de la Dixmerie écrit du Concert spirituel qu'il s'agit de «ce Spectacle où l'on peut jouir le plus complettement \& des meilleures 
symphonies de chaque nation, \& de la musique propre aux instrumens de toute espèce, \& de l'exécution des plus habiles Maîtres dans des genres si opposés ${ }^{25} » \ldots$

Impossible soumission à un programme national, dynamique profondément «transnationale » que Messiaen met en avant lorsqu'il évoque le Domaine musical dans ses entretiens avec Claude Samuel: «Le Domaine musical fut le lieu où l'on pouvait entendre les œuvres nouvelles qui venaient $d u$ monde entier (les très bonnes comme les moins bonnes - surtout les très bonnes! ${ }^{26} »$. Le fait fut parfois, si l'on en croit Jésus Aguila, mal ressenti par de jeunes compositeurs français de moindre renommée, peu joués au Domaine musical - considéré à cette aune comme "un centre d'importation exclusive des productions étrangères d'obédience sérielle ${ }^{27} »$. Claude Ballif aura alors ce mot : «Le Domaine musical est comme la Hanse : une coopérative d'import-export ${ }^{28}$ ", dont la connotation fortement commerciale ne doit probablement pas être reçue péjorativement tant sont palpables les logiques opérantes d'«échanges " internationaux, de "circulation» des œuvres ou de "marché commun» de la musique... toutes aussi bien ancrées dans un mouvement général de construction commerciale de l'Europe, mis en œuvre au sortir de la guerre afin d'assurer une paix durable.

Par la volonté des puissants, la paix des nations est fondée sur le commerce, tandis qu'au même moment le concert trouve sa raison d'être dans une forme de «commerce » et d'échange des œuvres. Or l'aspect foncièrement privé des origines du concert, tout en prononçant l'absence d'impulsion possible par un pouvoir, n'est pas étranger à l'évitement de la notion de frontière : le caractère privé de la chose échappe à toute mainmise d'un pouvoir - lequel s'exerce en de certaines limites. De fait, le pouvoir politique n'aura que très rarement eu l'initiative de séries de concerts. Il peut fonder des formations orchestrales ou des ensembles qui monteront inévitablement leurs saisons de concerts, mais de série de concert à part entière sans formation permanente, non. L'orchestre est un « objet » que l'on peut - ou croit pouvoir - saisir, le concert ne l'est pas. Le concert n'est pas saisissable - sinon de l'intérieur ${ }^{29}$ - et sa nécessité ne peut être, dans son mouvement initial, de conforter un pouvoir en exercice - ce qui peut en revanche parfois incomber à un orchestre, à une salle ou à une maison d'opéra. Il arrive cela étant, bien souvent, que l'initiative privée soit récupérée, adoubée ou épaulée, par la puissance dirigeante - le roi par l'intermédiaire de l'Académie royale ou l'État français via le ministère de la Culture -, répondant en particulier aux demandes de subsides (endémiques) ${ }^{30}$.

L'impossible soumission à des frontières tient donc tout à la fois d'un désir d'Europe, et d'une soustraction à la logique nationale : ni Boulez ni Philidor ne laissent voix à une restriction d'ordre national. Il est bien plutôt question de voir large, de voir au loin, autrement dit : il est fondamentalement question d'Europe ${ }^{31}$. Une Europe dont la figure se détache significativement dans le titre d'un recueil publié par Couperin en 1726, Les Nations, qui regroupe une série de sonates et de suites; chacun des quatre ordres réunis dans cet ensemble, intitulés La Françoise, L'Espagnole, L'Impériale et La Piémontaise, comprend des pièces écrites à la manière française et d'autres à la manière italienne. La contemporanéité de cette publication (1726) et de la naissance du Concert spirituel à Paris (1725) permet ici de comprendre le concert comme étant, à l'époque où le compositeur les conçoit, l'entreprise qui porte de telles rencontres entre les nations dans la cité. Autrement dit, le concert est l'inscription dans la cité d'une aspiration esthétique au dépassement des frontières. 

restriction programmatique. Bien qu'insaisissable par le pouvoir en place, elle joue par le fait d'une élite sociale et de classes dominantes proches de ce même pouvoir, restant systématiquement dans une marge relative... Ambivalence aussi peu négligeable que réductible : le concert existe dans cet interstice, au cœur même de la cité.

Ces toutes dernières lignes sciemment écrites au présent de l'indicatif sont le fait de la conviction sans preuve que le présent peut ici occuper sa valeur de vérité générale. Ceci n'est pas dire que tout concert peut ou doit répondre aux quelques observations que nous avons faites, mais la tentative d'imaginer un éventuel archétype - incomplet et discutable - à réinterroger. À tout le moins, accordons à ce temps et à ce mode une valeur d'analyse pour les deux cas étudiés dans ces lignes. Libre à nous de donner une suite à cette conception...

\section{BIBLIOGRAPHIE}

Aguila Jésus, Le Domaine musical. Pierre Boulez et vingt ans de création contemporaine, Paris, Fayard, 1992.

« Avertissement », Suite des divertissemens de Sceaux, Paris, E. Ganeau, 1725.

Bonnet George-Edgar, Philidor et l'évolution de la musique française au XVIII siècle, Paris, Librairie Delagrave, 1921.

Boulez Pierre, Correspondance, Winterthur, Amadeus Verlag, 1990.

Bricaire de la Dixmerie Nicolas, Lettres sur l'état présent de nos spectacles, Amsterdam/Paris, Duchesne, 1765.

Cessac Catherine, Portrait musical de la duchesse du Maine, Versailles, CMBV, 2003.

Dupont-Danican Philidor Jean-François, Dupont-Danican Philidor Nicolas, Les Philidor. Une dynastie de musiciens, Paris, Zurfluh, 1995.

Jamain Claude, L'Imaginaire de la musique au siècle des lumières, Paris, Honoré Champion, 2003.

Kaeppelin Virginie, André et Anne Danican Philidor. Ordinaires de la musique du Roi. Versailles 1687-1730, mémoire de maîtrise, université Paris IV Sorbonne, J. Gribenski (dir.), 1988.

La Borde Jean-Benjamin de, Essai sur la musique ancienne et moderne, t. III, chap. VIII, Paris, 1780.

" La musique contemporaine dans le "laboratoire" de J.-L. Barrault », Arts, 21 janvier 1954.

Langlois Frank, « Introduction », in Pierre Souvtchinsky, Un siècle de musique russe, Arles, Actes Sud, 2004.

Mozart Wolfgang Amadeus, Correspondance, Paris, Flammarion, 1987

Peyser Joan, Boulez. Composer, conductor, enigma, Londres, Cassell, 1976. 
Pierre Constant, Histoire du Concert spirituel, 1725-1790 [1900], Société française de musicologie, 1975.

Rougement Denis de, «Eur-opé comme "celle qui voit large, celle qui voit au loin" ", in Denis Guénoun, Hypothèses sur l'Europe, Belfort, Circé, 2000.

Samuel Claude, Permanences d'Olivier Messiaen, Arles, Actes Sud, 1999.

Souvtchinsky Pierre, «Ceux du Domaine musical », Cahiers Renaud-Barrault, n 41, décembre 1963.

\section{NOTES}

1. Pierre Souvtchinsky, "Ceux du Domaine musical », Cahiers Renaud-Barrault, $n^{\circ} 41$, décembre 1963, p. 144.

2. Constant Pierre, Histoire du Concert spirituel, 1725-1790 [1900], Société française de musicologie, 1975, p. 11.

3. Chacune de ces institutions a fait l'objet d'une monographie: l'Histoire du Concert spirituel, 1725-1790 de Constant Pierre a paru en 1900 et Le Domaine musical. Pierre Boulez et vingt ans de création contemporaine de Jésus Aguila en 1992 (Paris, Fayard).

4. Ces documents ont aujourd'hui pris l'eau dans les caves du Boulevard du Palais.

5. Souvtchinsky était, pour reprendre les mots de Frank Langlois, un «mécène sans argent. Sans argent, mais avec idées et réseaux qu'il dispensait sans limite à ceux qu'il avait élus » (introduction à P. Souvtchinsky, Un siècle de musique russe, Arles, Actes Sud, 2004, p. 13).

6. Lettre de Pierre Boulez à John Cage, juillet 1954 (Correspondance, Winterthur, Amadeus Verlag, 1990, p. 217).

7. Sur l'«arrangement » du piano par Cage, voir Joan Peyser, Boulez. Composer, conductor, enigma, Londres, Cassell, 1976, p. 61.

8. Présentation de la saison 1953-1954 par Pierre Boulez (texte introductif au programme de saison).

9. Ordinaire de la musique du roi à l'âge de 16 ans, Anne Danican Philidor devient Grand Hautbois de l'Écurie en 1698 et, en 1712, Dessus de Hautbois des Petits Violons de la Chambre (JeanFrançois et Nicolas Dupont-Danican Philidor, Les Philidor. Une dynastie de musiciens, Paris, Zurfluh, 1995, p. 36-39). Selon Constant Pierre, la charge de hautbois à la Chapelle date de 1704 (Histoire du Concert spirituel). George-Edgar Bonnet donne une chronologie légèrement différente (Philidor et l'évolution de la musique française au XVIII siècle, Paris, Librairie Delagrave, 1921, p. 23).

10. Extrait de l'acte passé le 22 janvier 1725. Dans Constant Pierre, Histoire du Concert spirituel, 1725-1790, p. 15.

11. Voir Claude Jamain, selon qui Marmontel était particulièrement sensible à cet aspect (L'Imaginaire de la musique au siècle des lumières, Paris, Honoré Champion, 2003, p. 56).

12. Voir Wolfgang Amadeus Mozart, Correspondance, Paris, Flammarion, 1987, lettres écrites par Léopold Mozart au printemps 1778.

13. Charles Burney comme Nicolas Bricaire de la Dixmerie écrivent pendant les dernières années du règne de Louis $\mathrm{XV}$. Voir infra, note 25.

14. Jean-François et Nicolas Dupont-Danican Philidor font à leur manière preuve de circonspection lorsqu'ils écrivent de leur aïeul qu'il est : «surintendant de la Musique du prince de Conti et sans doute Directeur des Concerts de la duchesse du Maine » (je souligne. Les Philidor. Une dynastie de musiciens, p. 39).

15. "Avertissement», Suite des divertissemens de Sceaux, Paris, E. Ganeau, 1725 (sans mention d'autorité). 
16. La période qui nous intéresse est située entre 1715 et 1725 . Or, après la mort du roi Louis XIV, La duchesse ne reprend l'organisation de fêtes à Sceaux qu'au terme de son emprisonnement par le régent, autour de 1720 .

17. Jean-Benjamin de La Borde, Essai sur la musique ancienne et moderne, t. III, chap. VIII, Paris, 1780 , p. 462.

18. Ce point resterait à éclaircir par un travail de recherches d'archives, les rares mentions faites de cet emploi n'étant jamais documentées. Virginie Kaeppelin note : «à partir de 1715, comme son père, Anne disparaît des minutes notariales laissant les musicologues désorientés. Sans doute, est-ce alors dans cette période qu'il fut nommé Surintendant de la musique du Prince de Conti et Directeur des Concerts de la Duchesse du Maine»-André et Anne Danican Philidor. Ordinaires de la musique du Roi. Versailles 1687-1730, mémoire de maîtrise, université Paris IV Sorbonne, J. Gribenski (dir.), 1988, p. 18. Mais l'abbé Genest, Adolphe Jullien, le général Piépape ou André Maurel, dans les ouvrages qu'ils ont consacrés à la « Reine de Sceaux », ne mentionnent jamais le nom du musicien, et Catherine Cessac dans son Portrait musical de la duchesse du Maine (Versailles, CMBV, 2003), plus particulièrement consacré à la question musicale, ne fait pas non plus la moindre allusion à son nom - quand bien même d'autres musiciens du roi sont cités.

19. "La musique contemporaine dans le "laboratoire" de J.-L. Barrault », Arts, 21 janvier 1954.

20. Boulez utilise alors l'expression «musicologie comparée» (programme de la saison 1953-1954).

21. Pierre Boulez. Propos recueillis par Jésus Aguila en 1988 (Le Domaine musical, p. 58).

22. Le Mercure de France, mars 1725, p. 616.

23. Voir George-Edgar Bonnet, Philidor et l'évolution de la musique française au XVIII siècle, p. 25-26.

24. À titre d'exemple, cette mention dans le Mercure de France de janvier 1723: «Ces fêtes magnifiques ont été embellies par des agrémens des Dames du pays, \& varié par des concerts de Musique Italienne \& Françoise. » (p. 191).

25. Je souligne. Nicolas Bricaire de la Dixmerie, Lettres sur l'état présent de nos spectacles, Amsterdam/Paris, Duchesne, 1765.

26. Je souligne. Claude Samuel, Permanences d'Olivier Messiaen, Arles, Actes Sud, 1999, p. 332.

27. Jésus Aguila, Le Domaine musical, p. 143.

28. Ibid.

29. C'est-à-dire : par les musiciens. Il n'est qu'à considérer l'usage de plus en plus fréquent, qui consiste à inviter un soliste à programmer "sa » série de concerts dans le cadre de la saison d'une salle ou d'un orchestre.

30. Il s'agit d'obtenir, pour le Concert spirituel, une diminution du montant de la redevance versée à l'Académie royale de musique et, pour le Domaine musical, des subventions de l'État. «Les divers détails de comptabilité que nous avons pu découvrir [...] démontrent l'impossibilité absolue pour un entrepreneur de tirer aucun bénéfice de son exploitation, les excédents de recettes étant constamment inférieurs à la redevance qu'imposait l'Opéra » écrit Constant Pierre dans son Histoire du Concert spirituel, p. 23.

31. Voir la reférence à l'étymologie établie par Denis de Rougement («Eur-opé comme "celle qui voit large, celle qui voit au loin" ») dans Denis Guénoun, Hypothèses sur l'Europe, Belfort, Circé, $2000, n^{\circ} 161$, p. 359. 


\section{RÉSUMÉS}

Dans un regard porté simultanément sur la naissance du Domaine musical au sortir de la deuxième guerre mondiale, et sur celle du Concert spirituel peu après la mort de Louis XIV, on se demande à quel point ces entreprises de concerts publics réguliers gardent, par-delà les siècles, une essence commune.

INDEX

Personnes citées : Philidor (Anne Danican), Boulez (Pierre)

Mots-clés : Concert spirituel, Domaine musical, Europe, histoire du concert

\section{AUTEUR}

\section{SARAH BARBEDETTE}

Étudiante en Littérature française et en Histoire de l'art à l'université Paris IV-Sorbonne, Sarah Barbedette prépare une thèse sous la direction de Denis Guénoun sur l'aspect visuel et scénique du concert. 